\title{
Análisis y Valoración del Paisaje como Patrimonio Cultural. El Caso del Valle de Ricote (Región de Murcia), Sureste, España
}

Analysis and assessment of the landscape as cultural heritage. The case of valle de ricote (region of Murcia), Southeast, Spain

Miguel Ángel Sánchez-Sánchez**

\section{RESUMEN}

A través del paisaje puede ser reconocido el patrimonio cultural, existente en el territorio, pudiéndosele atribuir al paisaje un carácter patrimonio-cultural. Indagar y reflexionar sobre esta cuestión constituye el objetivo de este trabajo. Mediante el análisis y valoración de los elementos que componen el paisaje, podemos acercarnos a un mejor conocimiento del carácter mencionado del paisaje. Para la valoración del paisaje, de sus elementos, son utilizados los distintos catálogos, listados, etc., donde estos son recogidos. Todo ello es reforzado con encuestas a residentes y no residentes, del territorio de estudio, donde se suministran una serie de ítems para ser valorados. La metodología ha sido aplicada al territorio del Valle de Ricote, en la Región de Murcia, claro ejemplo de un ámbito mediterráneo tradicional, con elevado potencial patrimonial y cultural. Existen elementos antrópicos de elevado valor -castillos, norias,..- así como naturales -espacios protegidos: Parques Naturales, RED NATURA 2000-. Esta cualidad de los mismos nos llevan a la conclusión de encontrarnos ante un paisaje sobre el que se puede afirmar tiene un carácter de patrimonio-cultural. El análisis y valoración de los elementos del paisaje permite saber el grado de valor como patrimonio cultural del mismo.

PALABRAS CLAVE: Paisaje, territorio, patrimonio, cultura, Valle de Ricote

\begin{abstract}
Through the landscape can be recognized the cultural heritage, existing in the territory, being able to attribute to the landscape a patrimony-cultural character. Inquiring and reflecting on this issue is the objective of this work. Through the analysis and evaluation of the elements that make up the landscape, we can get closer to a better knowledge of the mentioned nature of the landscape. For the evaluation of the landscape, of its elements, the different catalogs, lists, etc. are used, where they are collected. All this is reinforced with surveys of residents and non-residents of the study territory, where a series of items are supplied to be assessed. The methodology has been applied to the territory of the Ricote Valley, in the Region of Murcia, a clear example of a traditional Mediterranean environment, with a high patrimonial and cultural potential. There are anthropic elements of high value -castles, norias, ..- as well as natural -protected spaces: Natural Parks, RED NATURA 2000-. This quality of the same leads us to the conclusion of finding ourselves in front of a landscape that can be said to have a heritage-cultural character. The analysis and assessment of the elements of the landscape allows knowing the degree of value as cultural heritage of the same.
\end{abstract}

KEY WORDS: Landscape, territory, heritage, culture, Ricote Valley 


\section{INTRODUCCIÓN}

La definición de paisaje incluida en el Convenio Europeo del Paisaje firmado en el año 2000 [CEP2000], es tomada para este trabajo como premisa para estudiar las cuestiones relacionadas con el mismo. Cabe destacar dos aspectos recogidos en el artículo primero del CEP2000, la vinculación entre territorio y paisaje al ser entendido este último como cualquier parte del territorio... (SÁNCHEZ-SÁNCHEZ, 2017, p. 259) y el aspecto perceptivo de la población. En lo que respecta al territorio más allá de la función de soporte que puede ejercer (SOSA, 2012, p. 1), en el pueden desarrollarse interrelaciones entre elementos y procesos (SÁNCHEZ-SÁNCHEZ, 2017, p. 259). Estas vienen a ser lo que para el paisaje es la acción y la interacción de factores naturales y/o humanos, cuyo resultado es denominado como el carácter del mismo (CEP2000) (SÁNCHEZ-SÁNCHEZ, 2017, p. 259). Las huellas del territorio (CORBOZ, 1983, p. 34), como reflejo de las acciones e interrelaciones habidas sobre este, determinan/condicionan el carácter del paisaje (SÁNCHEZ-SÁNCHEZ, 2017, p. 259).

Diversos documentos institucionales y normativos atribuyen al paisaje la connotación patrimonial y/o patrimonio-cultural. Uno de los antecedentes, de donde parece apreciarse esta cuestión, es en la definición de Sitio Histórico contemplada en la Ley de Patrimonio Histórico Español de 1985, donde se entremezclan porción de territorio, sociedad, cultura y naturaleza (art.15) (CORTES GENERALES ESPAÑOLAS, 1985). La Carta del Paisaje Mediterráneo o Carta de Sevilla, reconoce el paisaje como patrimonio común a todos los individuos y sociedades (CONSEJO DE EUROPA, 1992). La creación del Plan Nacional de Paisaje Cultural servirá para la acción sobre paisajes relevantes por su significado cultural. En este Plan se afirma la conversión del paisaje en un tipo particular de patrimonio, aumentando su importancia cultural (MECD-IPCE, 2002). Algunas instituciones internacionales, Alianza de Paisajes Culturales Patrimonio Mundial, tal y como se puede apreciar, la importancia dada al paisaje como cultural y patrimonial es recogida en la denominación de tal institución (GOBIERNO DE ESPAÑA, 2017). Por último cabe hacer referencia a la Ley 42/2007, del Patrimonio Natural y de la Biodiversidad, en ella el paisaje natural es reconocido como un recurso natural (art.3.30), el paisaje como patrimonio natural (art.27), y del artículo 35 dedicado a la figura de "Paisaje Protegido" se desprende la 
connotación cultural atribuible al paisaje (CORTES GENERALES ESPAÑOLAS, 2007a).

En el ámbito de las comunidades autónomas españolas algunas normativas hacen referencia al paisaje como patrimonio cultural. La Ley 4/2004, de Ordenación del Territorio y Protección del Paisaje de la Comunidad Valenciana, en su preámbulo considera al paisaje como patrimonio común, pero no será hasta el art.70.a, donde se hará una alusión más directa a la cuestión patrimonio-cultural de este, al asociar al mismo y al territorio la existencia de un patrimonio cultural. Finalmente, en lo que respecta a esta ley, hay que hacer notar que a través del art. 86 se etiqueta al territorio y el paisaje como patrimonial (CORTES VALENCIANAS, 2004). Dicha ley será derogada por la Ley 5/2014, de Ordenación del Territorio, Urbanismo y Paisaje, de la Comunitat Valenciana, no contraviniendo lo anterior, y añadiendo que las áreas agrícolas conforman un paisaje cultural identitario (CORTES VALENCIANAS, 2014).

Otras normativas autonómicas le atribuyen valor patrimonial, histórico y socio-cultural, Ley catalana 8 de 2005 sobre la protección, gestión y ordenación del paisaje (PARLAMENT DE CATALUNYA, 2005). Cultural e histórico, Reglamento de Ordenación del Territorio y Urbanismo del Principado de Asturias (CONSEJO DE GOBIERNO DEL PRINCIPADO DE ASTURIAS, 2007). O como componente fundamental del patrimonio cultural, Ley 7/2008, de 7 de Julio, de protección del paisaje de Galicia (PARLAMENTO DE GALICIA, 2008). Por último cabe citar la Ley de Patrimonio Histórico de la Comunidad de Madrid, en la que se establece una categoría de Bien de Interés Cultural [BIC] denominada "Paisaje Cultural" (art.3) (ASAMBLEA DE MADRID, 2013).

En el ámbito de la Región de Murcia, el Atlas de los Paisajes de la Región de Murcia estima que los paisajes de regadío de huerta o vega son a la vez culturales y patrimoniales y suponen una pieza del patrimonio cultural (PRIETO, ET AL., 2009). Para la Estrategia del Paisaje de la Región de Murcia (CARM, 2011), el CEP2000 pone de manifiesto el innegable protagonismo del paisaje como elemento patrimonial. La Ley de Ordenación Territorial y Urbanística de la Región de Murcia [LOTURM] 13/2015 afirma que "La estrategia del Paisaje pretende reconocer el paisaje como expresión de la diversidad del patrimonio común cultural y natural" (ASAMBLEA REGIONAL DE MURCIA, 2015). 
Además algunos autores hacen referencia en sus trabajos a esa vertiente patrimonio-cultural del paisaje. Para Gómez-Ortíz, et al. (2010) la valorización social del patrimonio no es ajena al paisaje, en tanto en cuanto es tratado también como patrimonio. Rivera Blanco (2010, p. 22-23) afirma que el paisaje cultural se justifica por la fuerza de la asociación, de algunos fenómenos -entre ellos los culturales-, con el elemento natural. En este trabajo, entendemos el paisaje, como un fenómeno patrimonial de acuerdo con Rivera Blanco (2010, p. 11), con la calificación añadida de cultural, pues todo territorio está afectado por la acción del hombre. Mata Olmo (2008, p. 158, 162, 168) tras argumentar sobre la concepción patrimonial del paisaje, nos lleva un territorio de convergencia entre patrimonio y paisaje cultural a través de la gestión del primero. Zoido (2017) en alusión a los valores culturales del territorio y por ende del paisaje, recogidos en su trabajo sobre los paisajes como patrimonio natural y cultural, nos acerca a esa visión del paisaje como patrimonio cultural. Por último cabe mencionar la reflexión realizada por Gómez-Mendoza (2013) en su trabajo "Del patrimonio paisaje a los paisajes patrimonio" donde se reafirma la carácter patrimonial del mismo.

Conocer, saber sobre esa visión del paisaje como patrimonio cultural, resulta de interés. Los territorios contienen elementos y procesos, tanto naturales como antrópicos, que constituyen un patrimonio de los pueblos y sociedades que los habitan. Estos patrimonios están influenciados por la cultural de los grupos sociales. A su vez las peculiaridades geográficas del territorio, influye en esa cultural, dándose un proceso de intercambio, influyente, en un sentido y otro. Todo ello queda reflejado en los diversos paisajes producto de la percepción territorial. Por lo que el paisaje puede ser considerado como patrimonio cultural. Para indagar sobre este aspecto es necesario recurrir a una metodología que nos aproxime a un mejor conocimiento del mismo.

Ciertos autores para llevar a cabo una mejor comprensión del paisaje realizan una clasificación de los elementos del mismo, para su posterior análisis y valoración (FIDALGO, 2014, p. 11-27). Otros proponen la realización de un "Inventario Territorial" donde se incluye, entre sus tipologías de recursos, los histórico-culturales, ambientales, paisajísticos, y etnológico-antropológicos (TROITIÑO, 1998, p. 100-101) La realización de encuestas a la población es otra vía de exploración. Esta permite conocer la percepción que se tiene sobre el territorio y sus elementos. 
El Valle de Ricote en la Región de Murcia (Fig. 1) es una de esas áreas del ámbito mediterráneo donde se aúnan elementos y procesos territoriales con carácter histórico, cultural, identitario, etc., dando lugar a un paisaje con considerable valor patrimonial y cultural. Por lo que es tomado en este trabajo como referencia sobre el que aplicar la propuesta metodológica para el conocimiento de ese aspecto patrimonio-cultural del paisaje.

Se tiene como objetivo reflexionar e indagar para realizar una propuesta metodológica que permita analizar y valorar el paisaje como patrimonio cultural en ámbitos mediterráneos, en concreto en el Valle de Ricote.

Figura 1. Localización y límites del Valle de Ricote.

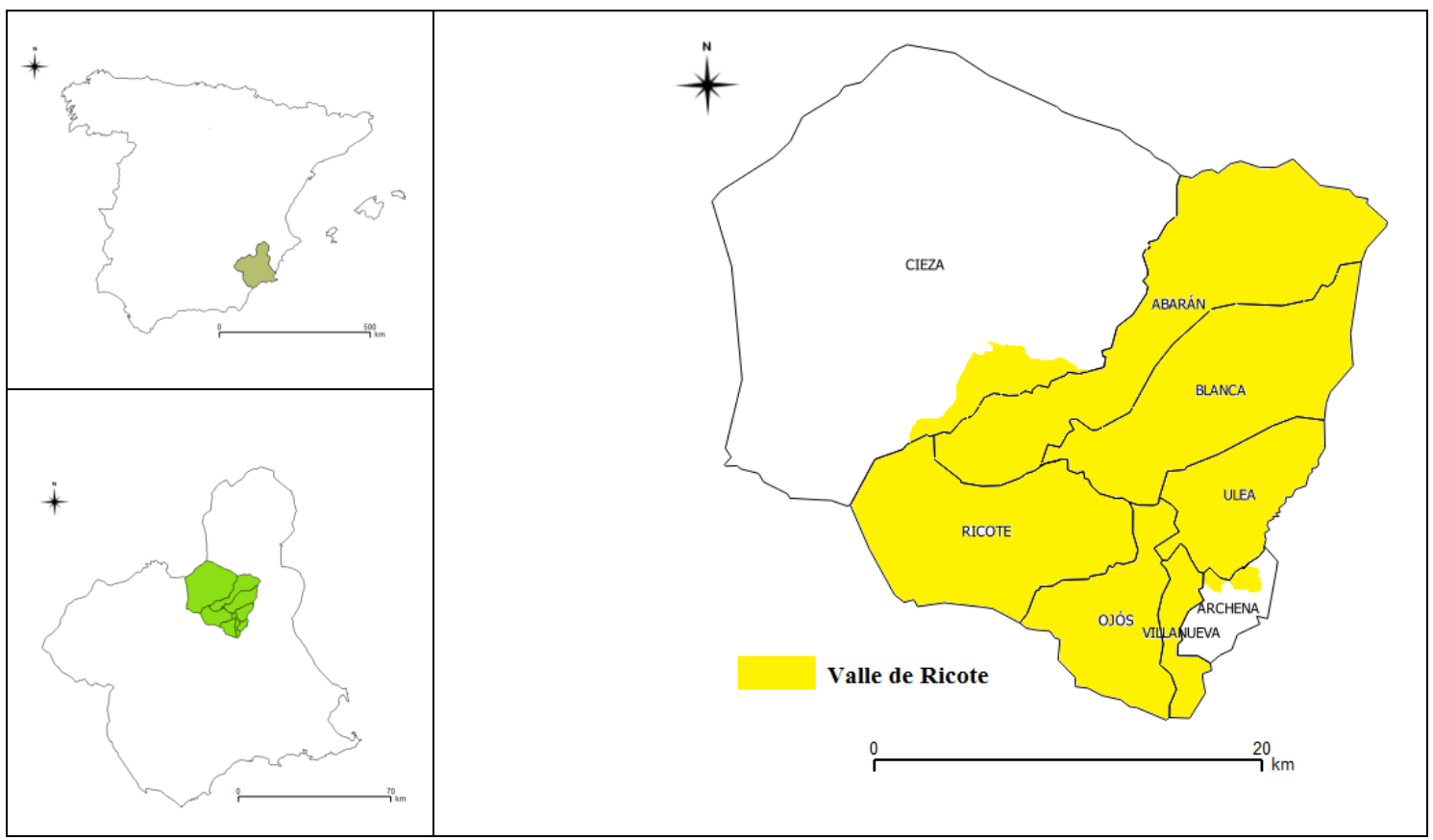

Fuente: Sánchez-Sánchez, et al. (2015).

\section{METODOLOGía}

Para abordar el análisis del paisaje previamente resulta adecuado realizar algunas consideraciones respecto a ese aspecto patrimonial del paisaje. Tal y como afirma López Martín (2006) el estudio del paisaje va unido intrínsecamente al hecho patrimonial. Por tanto en el análisis del paisaje hay que considerar dicha cuestión. Pero ¿cuál es el hecho patrimonial a analizar, de los existentes en el territorio?, dicho de otro modo: ¿qué patrimonio hay que estudiar? La idea de patrimonio ha ido evolucionando desde un planteamiento particularista, centrado en la propiedad privada y el disfrute 
individual (LLULL, 2005) hacia postulados más colectivistas. No obstante es pertinente realizar alguna observación al respecto, para superar esa visión algo constreñida. En nuestro caso encontramos patrimonios privativos, de titularidad particular, pero de disfrute colectivo, sirva como ejemplo el disfrute del paisaje y beneficios para el medio y la salud de los montes; o los cascos urbanos históricos donde existen elementos privados pero no por ello imposible de disfrutar de su aportación al conjunto urbano. No obstante a la hora de seleccionar los elementos de resaltada significación patrimonial, se tiene en cuenta el carácter colectivo, tal y como es recogido por Lull (2005, p. 180).

El análisis conlleva la distinción y separación de las partes de algo para conocer su composición (RAE, 2017). La valoración constituye el reconocimiento, estimación o apreciación del valor o mérito de algo o alguien (RAE, 2017). En aras a una mayor concreción, cabe decir, que para el desarrollo del análisis y valoración se indago sobre elementos y procesos existentes en el territorio con valor patrimonial y cultural.

Para el análisis y valoración de los elementos del paisaje, que pudieran contribuir a un mejor conocimiento de este como, patrimonio cultural, se tomó, como referencia la catalogación, de los mismos, realizada por Fidalgo (2014) adaptada para el caso del Valle de Ricote (Tabla 1).

Fueron tenidos en cuenta los componentes: antrópicos, bióticos y abiótiocos. $\mathrm{Su}$ caracterización permite perfilar sobre qué elementos del paisaje se trabajará:

1) Antrópicos. Son elementos de origen o existencia directamente relacionada con la actividad humana. Visibles, porque la tecnología ha permitido la alteración de las características originales del medio ambiente. Destacan sus contrastes, delimitados por líneas bien definidas y volúmenes simples, en oposición a las líneas más orgánicas y recortadas que caracterizan los componentes de origen natural.

2) Bióticos. Elementos vivos que pueden ser observados a simple vista sin instrumentos ópticos, como por ejemplo vegetales y animales.

3) Abióticos. Elementos de origen natural que determinan los atributos formales de la superficie de la tierra. Algunas de sus categorías utilizadas para la ocasión son: a) Formas líquidas. Son las adoptadas por el agua en estado líquido, y b) Formas sólidas. Conforman la superficie del territorio y le otorgan carácter (FIDALGO, 2014, p. 11-27). El paisaje en su dimensión patrimonial integra el natural y el cultural. En el campo del patrimonio natural están contenidos los sitios y la diversidad biológica. Para el caso del 
patrimonio cultural se tuvo en cuenta el patrimonio monumental (AYUSO et al., 2009, p. 28).

Para la valoración de los elementos con carácter patrimonial significativo se recurrió a los diferentes catálogos (CAPEL, 2014) existentes, con el fin de comprobar su inclusión o no en los mismos, grado de protección del elemento o proceso territorial, etc. Esto permitió estimar el valor de la importancia intrínseca de estos. Los catálogos, listados, etc., que se utilizaron son:

- Áreas y espacios naturales protegidos de la Comunidad Autónoma de la Región de Murcia, Red Natura 2000, etc.; para el caso del patrimonio natural.

- Censo de Bienes de Interés Cultural de la Región de Murcia (PATRIMUR-CARM, 2017), catálogos de bienes protegidos contemplados en los Planes Generales Municipales de Ordenación (PGMO).

- Normas Subsidiarias (NNSS).

- Páginas web de los ayuntamientos.

Tabla 1. Clasificación de los elementos que caracterizan el paisaje.

\begin{tabular}{|c|c|}
\hline \multicolumn{2}{|c|}{$\begin{array}{l}\text { ELEMENTOS QUE CARACTERIZAN EL PAISAJE } \\
\end{array}$} \\
\hline \multicolumn{2}{|c|}{ A.ANTRÓPICOS } \\
\hline \multicolumn{2}{|l|}{ A1.URBANOS } \\
\hline 1. Urbanización. & $\begin{array}{l}\text { 1.1. Construcciones arquitectónicas. } \\
\text { 1.2. Espacios públicos (parques, plazas, etc.). } \\
\text { 1.3. Plano urbano. }\end{array}$ \\
\hline 2. Vías de comunicación. & $\begin{array}{l}\text { 2.1. Funcionales. } \\
\text { 2.2. No funcionales. }\end{array}$ \\
\hline \multicolumn{2}{|l|}{ A2. AGRÍCOLAS } \\
\hline $\begin{array}{l}\text { 1. Áreas de actividad } \\
\text { agrícola. }\end{array}$ & $\begin{array}{l}\text { 1.1. Secano. } \\
\text { 1.2. Regadío. } \\
\text { 1.2.1. Nuevos. } \\
\text { 1.2.3. Tradicionales }\end{array}$ \\
\hline 2. Formas vegetales. & $\begin{array}{l}\text { 2.1. Dominancia (proporción del territorio ocupado } \\
\text { visualmente por una determinada especie -más } \\
\text { dominante/menos dominante-). }\end{array}$ \\
\hline 3. Construcciones. & $\begin{array}{l}\text { 3.1. Edificaciones. a) Viviendas. b) Otras. } \\
\text { 3.2. Infraestructuras }\end{array}$ \\
\hline 4. Vías de comunicación. & 4.1. Caminos rurales \\
\hline \multicolumn{2}{|l|}{ B. NATURALES } \\
\hline \multicolumn{2}{|l|}{ 1B. ABIÓTICOS } \\
\hline $\begin{array}{l}\text { 1. Formas líquidas (masas } \\
\text { de agua). }\end{array}$ & $\begin{array}{l}\text { 1. Formas de agua, superficiales terrestres. } \\
\text { 1.1. Puntual (fuentes, pozos, manantiales). } \\
\text { 1.2. Lineal (ríos). } \\
\text { 1.3. Plano (lagos, embalses). }\end{array}$ \\
\hline 2. Formas sólidas. & 2.1. Suelos. a) desiertos, acción del fuego, etc. \\
\hline
\end{tabular}




\begin{tabular}{|l|l|}
\hline & $\begin{array}{l}2.2 . \quad \text { Atributos geomorfológicos (según } \\
\text { observaciones de sus actuales formas y atributos). } \\
\text { a) Pendiente, b) Exposición, c) Altitud, d) } \\
\text { Elementos del suelo (pedregosidad, afloramientos } \\
\text { rocosos y depósitos de superficie). }\end{array}$ \\
\hline 2B. BIÓTICOS & $\begin{array}{l}\text { 1.1. Dominancia (proporción del territorio ocupado } \\
\text { visualmente por una determinada especie -más } \\
\text { 1. Fominante/menos dominante-). }\end{array}$ \\
\hline
\end{tabular}

Fuente: Fidalgo (2014).

Todo lo anterior fue complementado con la realización de encuestas-entrevistas, con el fin de obtener información sobre la percepción de los entrevistados frente a algunos elementos o/y hechos geográficos existentes en el área de estudio. Se les solicitó una valoración de los mismos. Tal valoración fue realizada mediante la técnica de las escalas de categorías, el sujeto evaluaba directamente la sensación, percepción, etc., hacia un ítem -suministrado previamente, tras una prospección documental y de campo para su selección-, empleando una escala con varias categorías de respuesta (DURÁN, et al., 2000, p. 30). La escala utilizada para la percepción, fue de las denominadas escalas numéricas, es decir, aquellas que permiten a los encuestados dar una nota para valorar los atributos. Esta es de las consideradas cortas, donde el valor más bajo se corresponde con máxima insatisfacción y el valor más alto con la máxima satisfacción (AYUNTAMIENTO DE MADRID, 2012, p. 36). Para ello se valoró de 0-4 $(0$ = nada interesante; $4=$ muy interesante $)$. Las encuestas fueron dirigidas tanto a los residentes en el área de estudio, como a los visitantes (Tabla 2).

Tabla 2. Encuesta de conocimiento y valoración de elementos materiales e inmateriales.

\begin{tabular}{|l|l|c|c|c|c|c|c|c||}
\hline $\begin{array}{l}\text { RESPONDA SI CONOCE USTED ESTOS ELEMENTOS Y VALORELOS } \\
\text { (0 NADA INTERESANTE; 4 MUY INTERESANTE) }\end{array}$ \\
\hline ELEMENTOS PATRIMONIALES & ¿CONOCE? & $\mathbf{0}$ & $\mathbf{1}$ & $\mathbf{2}$ & $\mathbf{3}$ & $\mathbf{4}$ \\
\hline SIERRA DE RICOTE & SI & NO & & & & & \\
\hline RIO SEGURA (VALLE DE RICOTE) & SI & NO & & & & & \\
\hline PICO DEL CAJAL & SI & NO & & & & & \\
\hline SIERRA DEL ORO & SI & NO & & & & & \\
\hline PUERTO DE LA LOSILLA & SI & NO & & & & & \\
\hline CRUCE DE ABARÁN & SI & NO & & & & & \\
\hline SIERRA DE LA PILA & SI & NO & & & & & \\
\hline AZUD DE OJÓS & SI & NO & & & & & \\
\hline EL SOLVENTE & SI & NO & & & & & \\
\hline SIYASA (CIEZA) & SI & NO & & & & & \\
\hline
\end{tabular}




\begin{tabular}{||l|c|c|c|c|c|c|c||}
\hline BALNEARIO DE ARCHENA & SI & NO & & & & \\
\hline CASA DEL CURA (ULEA) & SI & NO & & & & \\
\hline SALTO DE LA NOVIA & SI & NO & & & & \\
\hline IGLESIA SAN BARTOLOMÉ (ULEA) & SI & NO & & & & & \\
\hline EL GURUGÚ (ULEA) & SI & NO & & & & \\
\hline CASTILLO DE BLANCA & SI & NO & & & & \\
\hline FUENTE DEL COBI (VILLANUEVA) & SI & NO & & & & \\
\hline ENCIERROS DE BLANCA & SI & NO & & & & \\
\hline ERMITA STOS. MÉDICOS (ABARÁN) & SI & NO & & & & & \\
\hline ERMITA SAN ROQUE (BLANCA) & SI & NO & & & & & \\
\hline IGLESIA DE RICOTE & SI & NO & & & & & \\
\hline CASA DE LA ENCOMIENDA (RICOTE) & SI & NO & & & & & \\
\hline AYUNTAMIENTO DE RICOTE & SI & NO & & & & & \\
\hline FIESTAS VERA CRUZ ULEA & SI & NO & & & & & \\
\hline HOYA DEL CAMPO (ABARÁN) & SI & NO & & & & & \\
\hline ESTACIÓN BLANCA-ABARÁN & SI & NO & & & & & \\
\hline EL BOQUERON (ABARÁN) & SI & NO & & & & & \\
\hline LA BERMEJA (RICOTE) & SI & NO & & & & & \\
\hline BALNEARIO DE ARCHENA & SI & NO & & & & & \\
\hline NORIAS DE ABARÁN & SI & NO & & & & \\
\hline ALQUIBLA (VILLANUEVA) & SI & NO & & & & & \\
\hline \hline
\end{tabular}

Fuente: elaboración propia.

\section{RESULTADOS Y DISCUSIÓN}

En la margen derecha del río Segura, eje vertebrador del Valle (Fig. 2), se encuentra la Casa del Menjú incluida en el catálogo de bienes inmuebles y elementos protegidos de Cieza (CIEZA-PGMO, 2008). Esta se sitúa sobre una finca agro-forestal donde se alternan regadíos tradicionales, de valor etnográfico e histórico, con los nuevos cultivos arbóreos con riego localizado. Las infraestructuras hidráulicas, existentes en el municipio de Cieza, están incluidas en el mencionado catálogo (CIEZA-PGMO, 2008). En la actualidad se encuentra incoado expediente para la declaración de Bien de Interés Cultural [BIC], con la categoría de lugar de interés etnográfico, del territorio que discurre desde El Menjú hasta el puente de Hierro de Abarán declarado BIC. La propuesta, realizada por el Ayuntamiento de Abarán, de declaración de BIC, incluye elementos, de gran valor tales como las norias, acequias, azud, hormas de piedra, etc. (BORM, 2016).

En esta misma margen, del río, a una cota superior dominando el Valle, constituyéndose en la puerta norte de entrada al mismo, se localizan: el castillo de la Atalaya del siglo XII, despoblado islámico "Medina Siyâsa" (PATRIMUR-CARM, 
2017), ermita del Buen Suceso y pozo de nieve (CIEZA-PGMO, 2008). Sin abandonar el municipio ciezano, en la margen izquierda destaca la existencia de yacimientos arqueológicos en el paraje de Bolvax (Ibérico, Bronce tardío y final, y Paleolítico medio) declarados como BIC (PATRIMUR-CARM, 2017) (CIEZA-PGMO, 2008).

El Puente de Hierro de Abarán, ya citado anteriormente, ostenta la catalogación de BIC (PATRIMUR-CARM, 2017). Además del teatro Cervantes. Otros elementos de interés son los diversos yacimientos arqueológicos, entre ellos: El Molar, Cabezo del Judío y La Carrahila (ABARÁN-PGMO, 2016).

Figura 2. Vista área parcial del Valle de Ricote.

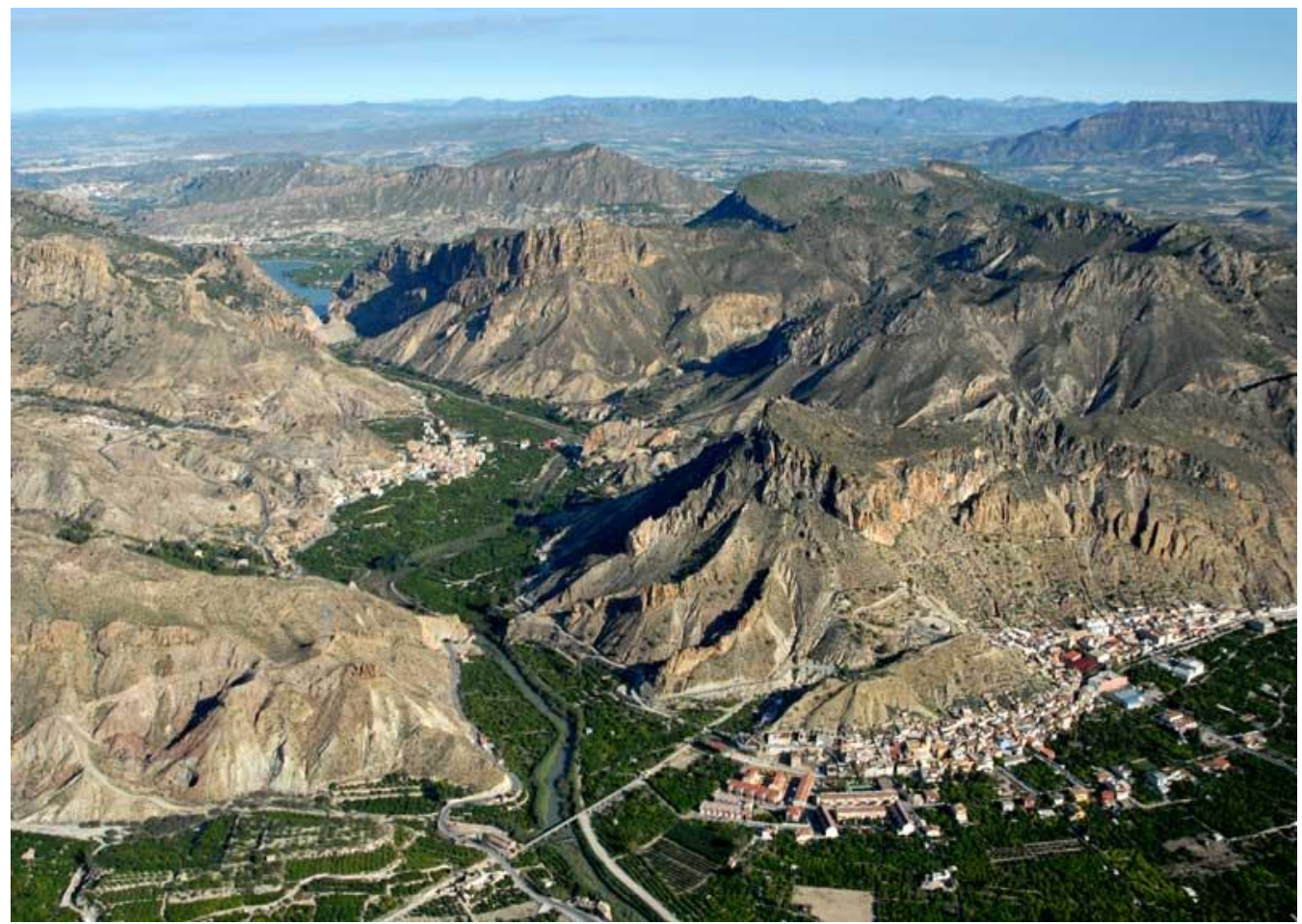

Fuente: Región de Murcia Digital (2017).

Entre los municipios de Abarán y Blanca se encuentra el yacimiento arqueológico Cabezo de la Cobertera (BIC) (PATRIMUR-CARM, 2017). Se trata de un cerro testigo, cuyos restos arqueológicos se identifican como un granero colectivo fortificado construido en época almohade (s. XIII d.C) (BORM, 2010).

Aguas abajo y en la localidad de Blanca el elemento del paisaje de carácter antrópico urbano destacable, es el casco urbano. A su vez, quizás el castillo (siglo XIII) es el elemento emblemático más sobresaliente. Declarado BIC junto a una serie de escudos existentes en la localidad (PATRIMUR-CARM, 2017). El catálogo de bienes protegidos de Blanca incluye el casco histórico de la población, así como un buen 
número de edificaciones entre las que se incluye la iglesia y el ayuntamiento así como una de las plazas de la localidad (BLANCA-PGMO, 2008). La antigua fábrica de la luz, es otra destacada edificación, musealizada actualmente, convertida en el Centro de Interpretación de La Luz y el Agua, junto al río Segura.

El elemento agrícola se definido por la existencia de las huertas tradicionales (Huerta de Abajo, Buila y Bayna), buena parte de estas sumergidas bajo el embalse. Entre las edificaciones existentes destaca La Favorita (villa de recreo del Conde de la Vallesa).

En lo que respecta a los elementos naturales, aunque de origen humano, destaca una de las formas líquidas abióticas que mayor singularidad da la zona. Se trata de la lámina de agua formada por el embalse de Blanca. En cuanto los elementos naturales bióticos junto a las palmeras datileras (Phoenix dactylifera), en las márgenes del embalse se encuentra el taray (Tamarix canariensis). De entre la especies de flora silvestre sobresale el rabogato rosado (Sideritis glauca) siendo una especie vulnerable. Todas ellas se encuentran incluidas en el catálogo regional de especies silvestres de flora protegida (BORM, 2003). Entre la fauna ornitológica están presentes entre otros la garza real, garceta común, garcilla bueyera, avetorillo común y martinete común (CABALLERO, 2007).

En la población de Ojós la iglesia de San Agustín es la única edificación catalogada como BIC. Además en el casco urbano ostentan tal consideración los escudos: de los Marín y los sitos en la calle Sargento Melgarejo y en el callejón de Massa (PATRIMUR-CARM, 2017). En el marco de otras catalogaciones se incluyen algunas construcciones más, tales como algunas casonas, ayuntamiento y lavadero público, siendo este último el mejor conservado en el Valle. Siendo quizás el elemento más singular del casco urbano (OJÓS-PGMO, 2014). Los sistemas agrícolas albergan un agrosistema de huerta tradicional, donde los cítricos a base de limoneros dominan sobre otras especies. También existen acequias que parten del azud de Ojós, el cual contribuye mediante el sangrado del rio a la existencia de las huertas del Ojós, Ulea y Villanueva. Algunas de las edificaciones de valor ambiental protegidas son: la noria de Ribera, del Olivar, de la Coya y del Solvente; Ceña del Escobero; y Molino de la acequia de Villanueva (OJÓS-PGMO, 2014). 
Junto al río y en las cercanías de Ojós destacan las terrazas fluviales, situadas a cotas superiores, como herencia del discurrir pretérito del Segura a una mayor cota. La iglesia de San Bartolomé, en Ulea, es el único elemento declarado como BIC (PATRIMUR-CARM, 2017) de entre los elementos locales urbanos. Los bienes catalogados como patrimoniales desde el ámbito municipal, situados en el casco urbano, son el conjunto histórico, donde se incluyen: la casa parroquial, casa de la condesa de Villar de Felices, Ayuntamiento, El Henchidor, así como diversas casas (ULEA-PGMO, 2012). Villanueva del río Segura cuenta con varios BIC, su iglesia de la Asunción y tres escudos (PATRIMUR-CARM, 2017).

Siguiendo con los elementos antrópicos como son las áreas agrícolas, estas se encuentran representadas por las huertas tradicionales de Ulea y Villanueva. Con predominio de cítricos, limoneros en especial, y algunos frutales de hueso en menor medida. Como infraestructuras asociadas a actividad agrícola, tenemos las acequias que discurren por ambas márgenes del río. Catalogados como bienes patrimoniales en el municipio de Ulea -acequia Mayor-, así como los artefactos hidráulicos: Noria de Doña Elisa Carrillo y Noria del Conde de Villar de Felices (ULEA-PGMO, 2012). Bordeando el pueblo de Villanueva discurre hacia Archena la acequia del mismo nombre.

La finca El Parque (Ulea) conforma un espacio de gran interés. Estando declarado como bien patrimonial, así como las edificaciones existentes y el conjunto de palmeras y otros árboles. Se trata de uno de los pocos lugares, del Valle, donde perduran elementos propios del bosque de ribera. Otros elementos destacables presentes son: la fábrica de La Luz, donde existe un azud, y los restos del castillo, ambos incluidos en el catálogo municipal (ULEA-PGMO, 2012).

En territorio del municipio de Archena, el cual queda incluido en el Valle de Ricote (SÁNCHEZ-SÁNCHEZ, et al., 2015) existen el conjunto urbano de Los Baños (hotel, termas, ermita y casino), incluido en el catálogo de bienes y yacimientos protegidos de Archena. En el mismo se contemplan zonas de protección arqueológica: Baños de Archena, Castillo del Ciervo, Cabezo del Tío Pío, Cabezo Blanco, Cabezo Redondo, Cabezo del Pino y los Cabezos Viejos (ARCHENA-NNSS, 2006).

Fuera de lo que podría considerarse estrictamente como valle, desde una óptica geomorfológica, encontramos otros elementos territoriales y paisajísticos de interés. En el asentamiento poblacional de Ricote se localizan el palacete de Los Llamas, sede 
actual del Centro Cultural, anteriormente sede del Ayuntamiento, y la iglesia de San Sebastián, ambos del siglo XVIII y estilo barroco, están, declarados BIC. Además otros elementos arquitectónicos con tal consideración son: escudo en la fachada de Los Hoyos, escudo en antiguo Ayuntamiento y escudo en casa Juliana la Melona (PATRIMUR-CARM, 2017). El palacio de la Encomienda del siglo XV es otro elemento a destacar. Algunos árboles forman parte del catálogo de protección de Ricote: Olivo (Olea europea) y Algarrobo (Certonia siliqua) (RICOTE-PGMO, 2008). La actividad agraria organizada en torno al regadío tradicional, a base de cítricos, conforma la huerta tradicional de Ricote. En la misma se encuentran elementos patrimoniales destacados: Ermita de Nuestra Señora de las Huertas que fue antigua mezquita, ya citada así en 1495.

La Casa de Los Forestales y la Casa de Peones Camineros, en la carretera que une Ulea con la autovía (A-30), se encuentran catalogadas como patrimoniales (ULEAPGMO, 2012). El puerto de La Losilla, donde se localiza la a A-30, ha constituido un lugar de paso de destacadas vías de comunicación: calzada romana de Carthago Nova a Complutum, incluida en el catálogo municipal de Ulea como patrimonio arqueológico (ULEA-PGMO, 2012); camino Real de Cartagena a Madrid; Cañada Real de Los Cabañiles, todo ello da lugar a que a partir de 1800 el puerto de La Losilla constituya un nudo histórico de comunicaciones (MOLINA, 2003; LÓPEZ, 2007; LÓPEZ, 2009; QUIJADA, 2010; SÁNCHEZ-SÁNCHEZ et al, 2015). En el mismo se encuentran algunos restos de la Torre del Puerto de la Losilla declarados como BIC (PATRIMURCARM, 2017) y la inclusión del paraje "Los Diegos de Ulea" como patrimonio paleontológico en el catálogo de patrimonio del ayuntamiento de Ulea (ULEA-PGMO, 2012). Junto al mismo destaca la "Venta Puñales" anterior al siglo XVIII.

Otros patrimonios de carácter natural existentes en el área de estudio son: Parque Regional de La Pila, se trata de un parque natural con la máxima figura de protección existente en la legislación regional al efecto. A este hay que sumar las figuras de protección de la red Natura2000 presentes en el Valle: Lugar de Importancia Comunitaria [LIC] sierra de Ricote y La Navela; LIC Yesos de Ulea; y las Zonas de Especial Protección para las Aves (ZEPA) sierra de Ricote y La Navela, así como sierra del Molino, Embalse del Quipar y Llanos del Cagitán. Los dos primeros en aplicación de la Directiva 92/43/CEE del Consejo de 21 de mayo de 1992 relativa a la 
Sánchez- Sánchez, M.A.

conservación de los hábitats naturales y de la fauna y flora silvestre. Y las ZEPA en aplicación de la Directiva 2009/147/CE del Parlamento Europeo y del Consejo de 30 de noviembre de 2009 relativa a la conservación de las aves silvestres (MURCIANATURAL-CARM, 2017) (Fig. 3).

Figura 3. Diversos elementos y paisajes del Valle de Ricote

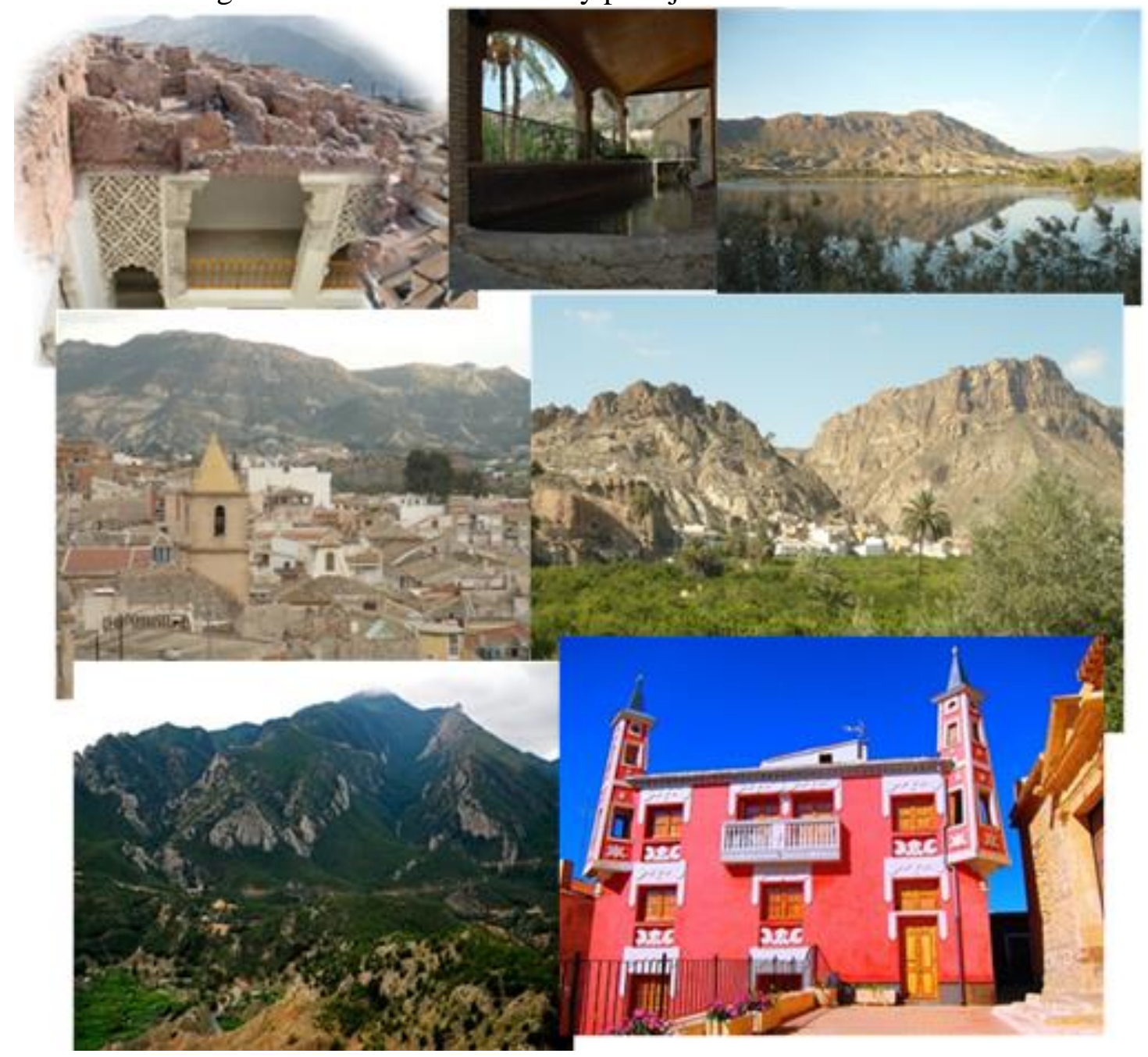

Fuente: Sánchez-Sánchez, et al. (2015).

De entre los resultados de las encuestas se han sombreado los máximos valores medios obtenidos, en función de la escala. Los residentes encuestados en la atribución de valores a los distintos ítems, sitúan el $67 \%$ de los mismos en el máximo valor (4), el $10 \%$ en el valor 3 y el $23 \%$ en el valor 2 (Tabla 3 ). 
Tabla 3. Resultados en tanto por ciento de las valoraciones de los RESIDENTES sobre diversos elementos del Valle de Ricote.

\begin{tabular}{|l|c|c|c|c|c|}
\hline \multirow{2}{*}{\multicolumn{1}{c|}{ ITEMS }} & \multicolumn{4}{|c|}{ ESCALA/VALORACIÓN } \\
\cline { 2 - 6 } & $\mathbf{0}$ & $\mathbf{1}$ & $\mathbf{2}$ & $\mathbf{3}$ & $\mathbf{4}$ \\
\hline SIERRA DE RICOTE & 1 & 1 & 12 & 26 & 60 \\
\hline RIO SEGURA (VALLE DE RICOTE) & 0 & 0 & 2 & 20 & 78 \\
\hline PICO DEL CAJAL & 0 & 6 & 42 & 27 & 25 \\
\hline SIERRA DEL ORO & 3 & 6 & 22 & 33 & 37 \\
\hline PUERTO DE LA LOSILLA & 8 & 19 & 36 & 15 & 22 \\
\hline CRUCE DE ABARÁN & 27 & 22 & 30 & 11 & 10 \\
\hline SIERRA DE LA PILA & 3 & 1 & 12 & 23 & 62 \\
\hline AZUD DE OJÓS & 0 & 0 & 11 & 29 & 59 \\
\hline EL SOLVENTE & 1 & 1 & 11 & 41 & 44 \\
\hline SIYASA (CIEZA) & 1 & 4 & 13 & 27 & 55 \\
\hline BALNEARIO DE ARCHENA & 0 & 1 & 8 & 14 & 77 \\
\hline CASA DEL CURA (ULEA) & 2 & 9 & 23 & 17 & 49 \\
\hline SALTO DE LA NOVIA & 0 & 4 & 20 & 24 & 51 \\
\hline IGLESIA SAN BARTOLOMÉ (ULEA) & 2 & 7 & 25 & 20 & 46 \\
\hline EL GURUGÚ (ULEA) & 4 & 8 & 21 & 28 & 40 \\
\hline CASTILLO DE BLANCA & 5 & 4 & 26 & 33 & 32 \\
\hline FUENTE DEL COBI (VILLANUEVA) & 6 & 13 & 33 & 15 & 33 \\
\hline ENCIERROS DE BLANCA & 10 & 8 & 15 & 22 & 45 \\
\hline ERMITA SANTOS MÉDICOS (ABARÁN) & 5 & 3 & 17 & 26 & 50 \\
\hline ERMITA SAN ROQUE (BLANCA) & 9 & 8 & 21 & 32 & 30 \\
\hline IGLESIA DE RICOTE & 2 & 3 & 12 & 27 & 56 \\
\hline CASA DE LA ENCOMIENDA (RICOTE) & 2 & 11 & 21 & 26 & 40 \\
\hline AYUNTAMIENTO DE RICOTE & 5 & 8 & 19 & 29 & 39 \\
\hline FIESTAS SANTISIMA VERA CRUZ ULEA & 0 & 8 & 18 & 26 & 48 \\
\hline HOYA DEL CAMPO (ABARÁN) & 16 & 23 & 32 & 16 & 13 \\
\hline ESTACIÓN BLANCA-ABARÁN & 17 & 25 & 27 & 16 & 16 \\
\hline EL BOQUERON (ABARÁN) & 4 & 12 & 31 & 30 & 22 \\
\hline LA BERMEJA (RICOTE) & 17 & 4 & 21 & 73 \\
\hline NORIAS DE ABARÁN & & 31 & 24 & 21 \\
\hline ALQUIBLA (VILLANUEVA) & & & \\
\hline
\end{tabular}

Fuente: elaboración propia.

En la tabla 4 se recogen los ítems cuyo máximo porcentaje de valoración se sitúa en el máximo valor (4) de la escala. A su vez estos fueron ordenados en función del mayor porcentaje obtenido estableciéndose un orden de mayor a menor. El elemento con mayor valoración es el río Segura, verdadero eje vertebrador del Valle. 
Tabla 4. Resultados de las opiniones de los RESIDENTES, en tanto por ciento, ordenados en función del mayor valor de la escala (4).

\begin{tabular}{|l|c|}
\hline \multicolumn{1}{|c|}{ ITEMS } & $\mathbf{4}$ \\
\hline RIO SEGURA (VALLE DE RICOTE) & 78 \\
\hline BALNEARIO DE ARCHENA & 77 \\
\hline NORIAS DE ABARÁN & 73 \\
\hline SIERRA DE LA PILA & 62 \\
\hline SIERRA DE RICOTE & 60 \\
\hline AZUD DE OJÓS & 59 \\
\hline IGLESIA DE RICOTE & 56 \\
\hline SIYASA (CIEZA) & 55 \\
\hline SALTO DE LA NOVIA & 51 \\
\hline ERMITA SANTOS MÉDICOS (ABARÁN) & 50 \\
\hline CASA DEL CURA (ULEA) & 49 \\
\hline FIESTAS SANTISIMA VERA CRUZ ULEA & 48 \\
\hline IGLESIA SAN BARTOLOMÉ (ULEA) & 46 \\
\hline ENCIERROS DE BLANCA & 45 \\
\hline EL SOLVENTE & 44 \\
\hline CASA DE LA ENCOMIENDA (RICOTE) & 40 \\
\hline EL GURUGÚ (ULEA) & 40 \\
\hline AYUNTAMIENTO DE RICOTE & 39 \\
\hline SIERRA DEL ORO & 37 \\
\hline FUENTE DEL COBI (VILLANUEVA) & 33 \\
\hline & \\
\hline
\end{tabular}

Fuente: elaboración propia.

En el caso de los no residentes los ítems distribuyen sus máximos porcentajes entre los valores 1, 2, 3 y 4 (Tabla 5) sin embargo en el caso de los residentes se distribuyen entre los valores 2, 3 y 4. Así a priori aparenta una mayor valoración de los ítems propuestos por parte de los residentes frente a los no residentes. Los porcentajes para la tabla 5 son: $18 \%, 25 \% ; 14 \%$ y $25 \%$ respectivamente con los valores $1,2,3$ y 4.

Tabla 5. Resultados en valores absolutos de las valoraciones de los NO RESIDENTES sobre diversos elementos del Valle de Ricote.

\begin{tabular}{|l|c|c|c|c|c|}
\hline \multirow{2}{*}{ ITEMS } & \multicolumn{5}{|c|}{ ESCALA/VALORES } \\
\cline { 2 - 6 } & $\mathbf{0}$ & $\mathbf{1}$ & $\mathbf{2}$ & $\mathbf{3}$ & $\mathbf{4}$ \\
\hline SIERRA DE RICOTE & 0 & 0 & 9 & 49 & 32 \\
\hline
\end{tabular}




\begin{tabular}{|l|c|c|c|c|c|} 
RIO SEGURA (VALLE DE RICOTE) & 0 & 0 & 15 & 32 & 56 \\
\hline PICO DEL CAJAL & 0 & 3 & 18 & 7 & 10 \\
\hline SIERRA DEL ORO & 0 & 3 & 32 & 20 & 10 \\
\hline PUERTO DE LA LOSILLA & 2 & 18 & 15 & 10 & 5 \\
\hline CRUCE DE ABARÁN & 15 & 27 & 9 & 10 & 2 \\
\hline SIERRA DE LA PILA & 0 & 6 & 24 & 29 & 37 \\
\hline AZUD DE OJÓS & 0 & 0 & 9 & 15 & 63 \\
\hline EL SOLVENTE & 0 & 0 & 18 & 12 & 24 \\
\hline SIYASA (CIEZA) & 0 & 3 & 0 & 29 & 32 \\
\hline BALNEARIO DE ARCHENA & 0 & 3 & 18 & 17 & 49 \\
\hline CASA DEL CURA (ULEA) & 0 & 3 & 9 & 15 & 17 \\
\hline SALTO DE LA NOVIA & 0 & 0 & 21 & 17 & 24 \\
\hline IGLESIA SAN BARTOLOMÉ (ULEA) & 0 & 3 & 9 & 15 & 20 \\
\hline EL GURUGÚ (ULEA) & 0 & 0 & 12 & 22 & 10 \\
\hline CASTILLO DE BLANCA & 0 & 6 & 18 & 17 & 15 \\
\hline FUENTE DEL COBI (VILLANUEVA) & 2 & 6 & 15 & 5 & 7 \\
\hline ENCIERROS DE BLANCA & 5 & 9 & 30 & 15 & 5 \\
\hline ERMITA SANTOS MÉDICOS (ABARÁN) & 0 & 6 & 12 & 7 & 7 \\
\hline ERMITA SAN ROQUE (BLANCA) & 0 & 3 & 18 & 5 & 10 \\
\hline IGLESIA DE RICOTE & 0 & 6 & 12 & 12 & 22 \\
\hline CASA DE LA ENCOMIENDA (RICOTE) & 0 & 3 & 3 & 12 & 20 \\
\hline AYUNTAMIENTO DE RICOTE & 0 & 9 & 15 & 12 & 17 \\
\hline FIESTAS STA. VERA CRUZ (ULEA) & 2 & 3 & 0 & 17 & 12 \\
\hline HOYA DEL CAMPO (ABARÁN) & 15 & 18 & 15 & 7 & 2 \\
\hline ESTACIÓN BLANCA-ABARÁN & 12 & 35 & 12 & 7 & 5 \\
\hline EL BOQUERON (ABARÁN) & 2 & 15 & 12 & 7 & 2 \\
\hline LA BERMEJA (RICOTE) & 0 & 3 & 6 & 15 & 7 \\
\hline NORIAS DE ABARÁN & 0 & 3 & 18 & 32 & 41 \\
\hline ALQUIBLA (VILLANUEVA) & 0 & 9 & 3 & 5 & 5 \\
\hline
\end{tabular}

Fuente: elaboración propia.

En la tabla 6 quedan recogidos aquellos ítems que fueron valorados con el valor más alto (4), por parte de los no residentes, y ordenados de mayor a menor tal y como se recoge.

Tabla 6. Resultados en tanto por ciento de las valoraciones de los NO RESIDENTES sobre diversos elementos del Valle de Ricote.

\begin{tabular}{|l|c|}
\hline \multicolumn{1}{|c|}{ ITEMS } & $\mathbf{4}$ \\
\hline AZUD DE OJÓS & 63 \\
\hline RIO SEGURA (VALLE DE RICOTE) & 56 \\
\hline NORIAS DE ABARÁN & 41 \\
\hline
\end{tabular}




\begin{tabular}{|l|c|} 
SIERRA DE LA PILA & 37 \\
\hline EL SOLVENTE & 24 \\
\hline IGLESIA DE RICOTE & 22 \\
\hline CASA DE LA ENCOMIENDA (RICOTE) & 20 \\
\hline AYUNTAMIENTO DE RICOTE & 17 \\
\hline
\end{tabular}

Fuente: elaboración propia.

Al comparar los datos obtenidos tanto para los residentes como no residentes se observó que los primeros situaron en el máximo valor 20 items sobre 28 (total ítems suministrados) (71\%). Por el contrario los no residentes sólo lo hicieron con 8 sobre 28 (29\%). Los valores medios de las tablas 4 y 6 , es respectivamente de 52 у 35. Centrándonos sólo en las tablas 20 y 22 se observa que el porcentaje de encuestados que daban en sus valoraciones en el nivel 4 de la escala, son sustancialmente mayores en los residentes, 78 y 77 para el primero y el segundo del ranking, que en los no residentes 63 y 56. Otro aspecto a resaltar que el río es considerado con el máximo valor y el mayor porcentaje de encuestados, 78, entre los residentes, y bajando al segundo lugar con un 63 , por parte de los no residentes.

\section{CONCLUSIONES}

Durante el análisis de los elementos del territorio, a la postre del paisaje, se ha podido comprobar que existe una extensa gama de los mismos. Tomar como base la metodología utilizada por Fidalgo para el análisis de los elementos que caracterizan el paisaje, constituye un marco adecuado. Permitiendo avanzar en la indagación y profundizar en el conocimiento de los elementos constitutivos del paisaje.

Nos situamos ante la existencia de elementos individuales, tangibles e intangibles, interelacionados entre sí, lo cual nos lleva hacia lo sistémico. Los elementos en ocasiones representan una síntesis de los aspectos sistémicos.

Durante el análisis se estudian los elementos del paisaje y no tanto la cuestión sistémica que puede suscitar el paisaje. Todos los elementos del paisaje, materiales e inmateriales, pueden ser considerados como patrimoniales, así como reflejo cultural, del momento, del lugar y de la sociedad. Mediante el análisis el paisaje es desmenuzado, en sus elementos, los cuales tras su estudio y valoración nos permiten conformarnos una idea del paisaje como patrimonio cultural. Indagar sobre cuestiones culturales y patrimoniales de los elementos, permitirá una valoración de los mismos. 
Dentro del marco de esta reflexión, para avanzar en una propuesta metodológica, resulta acertado utilizar uno o varios criterios discriminantes, con el fin de seleccionar aquellos elementos significativos. El valor que la sociedad le dé a los mismos, a través de los distintos catálogos, perfectamente puede constituir un adecuado criterio. El mismo es reforzado con la realización de encuestas, residentes y no residentes, para la obtención de una valoración sobre determinados ítems suministrados previamente.

El territorio escogido es un claro ejemplo de ámbito mediterráneo tradicional, considerado a priori con destacado valor cultural y patrimonial. Tras el análisis y valoración de los elementos del paisaje del Valle de Ricote, se puede afirmar que el paisaje en el Valle puede ser visto como patrimonio cultural. Esto es corroborado por la existencia de elementos individuales tangibles con destacado valor patrimonio-cultural, tanto de carácter antrópico como natural.

Indagar, reflexionar y avanzar en la vertiente sistémica del paisaje como contribuyente al reconocimiento patrimonio-cultural del paisaje puede ser una línea futura de interés para investigación en ámbitos mediterráneos.

\section{REFERENCIAS}

ABARÁN-PGMO, 2016. Ayuntamiento de Abarán-Plan General Municipal de Ordenación [PGMO].

ARCHENA.ES, 2016. Arqueología de Archena. [Consulta: 29 de octubre de 2017]. Disponible en Web: www.archena.es

ARCHENA-NNSS, 2006. Ayuntamiento de Archena-Normas subsidiarias de planeamiento [NNSS].

ASAMBLEA REGIONAL DE MURCIA, 2015. Ley 13/2015, de 30 de marzo, de ordenación territorial y urbanística de la Región de Murcia. Boletín Oficial de la Región de Murcia, no 77 de 6 de Abril de 2015.

AYUSO ÁLVAREZ, A.M., et al., 2009. Cultural, patrimonio y sostenibilidad. En Jiménez Herrero, Luís M. (Dir.). Patrimonio natural, cultural y paisajístico. Claves para la sostenibilidad territorial. Ed. Observatorio de la Sostenibilidad en España, Alcalá de Henares, p. 385.

AYUNTAMIENTO DE MADRID. Servicio de Evaluación. Subdirección General de Calidad y Evaluación , 2012. Criterios de orientación para la realización de encuestas de satisfacción. Metodología aprobada por decreto de 21 de diciembre de 2011 del Delegado del Área de Gobierno de Hacienda y Administración Pública. Madrid. 
BORM, 2003. Decreto $n^{\circ} 50 / 2003$, de 30 de mayo por el que se crea el Catálogo Regional de Flora Silvestre Protegida de la Región de Murcia y se dictan normas para el aprovechamiento de diversas especies forestales. Boletín Oficial de la Región de Murcia [BORM], $\mathrm{n}^{\circ} 131$, de 10 de Junio.

BORM, 2010. Resolución de 8 de noviembre de 2010 de la Dirección General de Bellas Artes y Bienes Culturales, por la que se incoa expediente de declaración de bien de interés cultural, con categoría de monumento, a favor del Cabezo de la Cobertera de Abarán y Blanca (Murcia). Boletín Oficial de la Región de Murcia [BORM], n 269, de 20 de Noviembre.

BORM, 2016. Resolución de 29 de junio de 2016 de la Dirección General de Bienes Culturales por la que se incoa procedimiento de declaración de bien de interés cultural, con la categoría de lugar de interés etnográfico, a favor de Las Norias de Abarán, en los términos municipales de Abarán y Cieza. Boletín Oficial de la Región de Murcia [BORM], nº 163, de 15 de Julio.

CABALLERO SOLER, J., 2007. "El paisaje antrópico del Azud de Ojós (Ojós-Blanca) Murcia. En Gómez Molina; Carrasco Molina (Coords.), Despierta tus sentidos (4 ${ }^{\circ}$ Congreso Internacional Valle de Ricote), Consorcio Turístico Mancomunidad "Valle de Ricote", 19-33.

CAPEL SÁEZ, H., 2014. El patrimonio: construcción del pasado y del futuro. Ediciones del Serbal, España, pp. 182.

CARM, 2011. Estrategia del paisaje de la Región de Murcia. Comunidad Autónoma de la Región de Murcia [CARM] http://www.sitmurcia.es/paisaje/ [consulta: 5 de Octubre de 2017].

CIEZA-PGMO, 2008. Ayuntamiento de Cieza-Plan General Municipal de Ordenación [PGMO].

CONSEJO DE EUROPA, 1992. Carta del paisaje Mediterráneo. (Carta de Sevilla). http://www.catpaisatge.net/fitxers/docs/convenis/Carta_Sevilla_Paisaje.pdf [consulta: 5 de Octubre de 2017].

CONSEJO DE GOBIERNO DEL PRINCIPADO DE ASTURIAS, 2007. Decreto 278/2007, de 4 de diciembre, por el que se aprueba el Reglamento de Ordenación del Territorio y Urbanismo del Principado de Asturias. Boletín Oficial del Principado de Asturias y de la Provincia, nº 38, de 15 de Febrero de 2008.

CORBOZ, ANDRÉ, 1983. "El territorio como palimpsesto". En Ángel Martín Ramos (ed.) Lo Urbano en 20 autores contemporáneos. Universitat Politécnica de Catalunya, 25-34.

CORTES GENERALES ESPAÑOLAS, 1985. Ley 16/1985, de 25 de junio, del Patrimonio Histórico Español. BOE, núm.155, de 29 de junio de 1985. 
CORTES GENERALES ESPAÑOLAS, 2007a. Ley 42/2007, de 13 de diciembre, del Patrimonio Natural y de la Biodiversidad. BOE, Num.299.

CORTES VALENCIANAS, 2004. Ley 4/2004, de 30 de junio, de la Genaralitat, de Ordenación el Territorio y Protección del Paisaje. Diari Oficial de la Comunitat Valenciana, núm. 4788.

CORTES VALENCIANAS, 2014. Ley 5/2014, de 25 de julio, de la Generalitat, de Ordenación del Territorio, Urbanismo y Paisaje, de la Comunitat Valenciana. Diari Oficial de la Comunitat Valenciana, Num.7329/31.07.2014.

DURÁN, A., OCAÑA, A.C., CAÑADAS, I. Y PÉREZ SANTAMARÍA, F., 2000. Construcción de cuestionarios para encuestas: el problema de la familiaridad de las opciones de respuesta. Metodología de Encuestas, Vol 2, Num1, 27-60.

FIDALGO, P., 2014. "Aportaciones para la definición de elementos visualesdeterminantes del paisaje". Cuadernos de investigación urbanística. Año VII, Núm.92, enero-febrero, pp. 1-92.

GOBIERNO DE ESPAÑA, 2017. Organismos e instituciones relacionadas con los paisajes culturales. http://www.mecd.gob.es [consulta: 13 de Octubre de 2017].

GÓMEZ ORTIZ, A., OLIVA-FRANGANILLO, M.; SALVÁ-CATARINEU, M., SALVADOR-FRANCH, F., 2010. El paisaje como valor patrimonial en los espacios protegidos: el caso del Parque Nacional de Sierra Nevada (España). Scripta Nova, Vol.XIV, 346

GÓMEZ-MENDOZA, J., 2013. Del patrimonio paisaje a los paisajes patrimonio. Documents d’Anàlisi Geográfica, vol. 59/1, 5-20. DOI: https://doi.org/10.5565/rev/dag.48

LÓPEZ MARTÍN, E., 2006. Criterios. Patrimonio y paisaje. Proyecto en el litoral malagueño. PH Boletín del Instituto Andaluz del Patrimonio Histórico, No 57, febrero, 97-108. DOI: https://doi.org/10.33349/2006.57.2147

LÓPEZ MORENO, J.J., 2007. La calzada Carthago Nova-Complutum y sus enlaces con el Valle de Ricote. En M ${ }^{\mathrm{a}}$ Cruz Gómez Molina y José Carrasco Molina (coord.), $4^{\circ}$ Congreso internacional Valle de Ricote "Despierta tus sentidos", Consorcio Turístico Mancomunidad "Valle de Ricote", 339-352.

LÓPEZ MORENO, J.J., 2009. La Cañada Real de los Cabañiles o de La Mancha a Murcia, una de las principales rutas prehistóricas en el sureste peninsular. Programa de festejos de Abarán, Edt. Ayuntamiento de Abarán.

LLULL PEÑALBA, J., 2005. "Evolución del concepto y de la significación social del patrimonio cultural". Arte, Individuo y Sociedad, 17:175-204. 
MATA OLMO, R., 2008. "El paisaje, patrimonio y recurso para el desarrollo territorial sostenible. Conocimiento y acción pública". Arbor, Ciencia, Pensamiento y Cultura, $\mathrm{N}^{\mathrm{o}}$ 729, 155-172. DOI: https://doi.org/10.3989/arbor.2008.i729.168

MECD-IPCE, 2002. Plan Nacional de Paisaje Cultural. Ministerio de Educación, Cultura y Deportes-Instituto de Patrimonio Cultural de España [MECD-PCE]. Madrid. MOLINA TEMPLADO, J.D., 2003. Los caminos del Valle en el pasado. En M $^{\text {a }}$ Cruz Gómez Molina y José Sánchez Ortíz de Villajos (coord.), II Congreso Turístico Cultural del Valle de Ricote "Despierta tus sentidos", Consorcio Turístico Mancomunidad "Valle de Ricote", 331-348.

MURCIANATURAL-CARM, 2017. Espacios protegidos. MURCIANATURALCARM [Comunidad Autónoma de la Región de Murcia]. http://www.murcianatural.carm.es [consulta: 5 de noviembre de 2017].

PARLAMENT DE CATALUNYA, 2005. Ley 8/2005, de 8 de junio, de protección, gestión y ordenación del paisaje de Cataluña. Diari Oficial de la Generalitat de Catalunya, Núm. 4407 de 16 de Junio de 2005.

PARLAMENTO DE GALICIA, 2008. Ley 7/2008, do 7 de julio, de protección da paisaje de Galicia. Diario Oficial de Galicia, No 139, de 18 de julio de 2008.

PATRIMUR-CARM，2017. Portal del patrimonio de la Región de Murcia [PATRIMUR]-Comunidad Autónoma de la Región de Murcia [CARM] [En línea]. [Consulta: 24 Octubre de 2017]. Disponible en Web: www.patrimur.es.

PRIETO CERDÁ, A.; FERNÁNDEZ MUÑOZ, S.C.; SANCHO URÍOS, J.C. (Coords.), 2009. Atlas del paisaje de la Región de Murcia. Consejería de Obras Públicas y Ordenación del Territorio de la Comunidad Autónoma de la Región de Murcia.

QUIJADA GUILLAMÓN, H.M., 2010. Dominio público, medio natural y rural en Abarán a través de las vías pecuarias. En Jesús Joaquín López Moreno (coord.), I Jornadas de Investigación y Divulgación sobre Abarán, pp. 187-203.

RICOTE-PGMO (2008). Ayuntamiento de Ricote-Plan General Municipal de Ordenación [PGMO].

SÁNCHEZ-SÁNCHEZ, M.A.; GARCÍA MARÍN, R.; BELMONTE SERRATO, F., 2015. "El Valle de Ricote en la Región de Murcia como recurso patrimonial e identidad cultural". En Análisis espacial y representación geográfica: innovación y aplicación, de la Riva, J. et al. (Eds.). Zaragoza: Universidad de Zaragoza-AGE, 1211-1219.

SÁNCHEZ-SÁNCHEZ, MIGUEL ÁNGEL, 2017. «Marco Teórico-Referencial para la protección, gestión y ordenación del paisaje. El Caso de la Región de Murcia», Espacio, Tiempo y Forma, serie VI Geografía 10 (2017), pp. 257-280. DOI: http://dx.doi.org/10.5944/etfvi.10.2017.19104 
SOSA, M., 2012. “¿Cómo entender el territorio?” 1ª Edición. Editorial Cara Parens. Universidad Rafael Landívar. Guatemala. 131 p. Colección Documentos para el debate y la formación, No. 4.

TROITIÑO VINUESA, M.A., 1998. "Patrimonio arquitectónico, cultura y territorio". Ciudades:Revista del Instituto de Urbanística de la Universidad de Valladolid. № 4, pp. 95-104.

ULEA-PGMO, 2012. Ayuntamiento de Ulea-Plan General Municipal de Ordenación [PGMO].

ZOIDO NARANJO, F., 2017. Los paisajes como patrimonio natural y cultural. I Congreso Internacional "El patrimonio cultural y natural como motor de desarrollo: investigación e innovación" / coord.por A. Peinado Herreros, 2012, p. 626-644. Disponible en: http://paisajeyterritorio.es/assets/los-paisajes-como-patrimonio-naturaly-cultural.-zoido-naranjo\%2C-f.pdf [consulta: 14 de octubre de 2017] 